\title{
PARTIAL TEXTURE ANALYSIS
}

\author{
H. J. BUNGE \\ Department of Physical Metallurgy, TU Clausthal, FRG
}

(Received 17 February 1989)

The texture is the orientation distribution function of the crystallites of a polycrystalline sample. Being a continuous function of three variables, it requires a large number of values for its complete description. The texture can be expressed in terms of a series expansion. It then requires a large number of coefficients for its complete description. When all functional values or all coefficients are determined from experimental measurements we speak of complete texture analysis. The most important methods for complete texture analysis are individual orientation measurements of a large number of crystals and pole figure measurement followed by pole figure inversion.

If only a limited number of values of the texture function or a few of its coefficients are being determined we speak of "partial texture analysis". The most important methods of partial texture analysis are the fixed angle texture analyzer and the measurement of the anisotropy of physical properties such as Young's modulus magnetic properties, thermal expansion and others.

KEY WORDS Pole figure measurement, individual orientation measurement, fixed angle texture analyzer, anisotropic properties.

\section{INTRODUCTION}

The texture of a polycrystalline material is defined as the orientation distribution function of the crystallites.

$$
f(g)=\frac{d V / V}{d g}, \quad g=\left\{\varphi_{1}, \phi, \varphi_{2}\right\}
$$

Thereby $d V / V$ is the volume fraction of crystals having the orientation $g$ of their crystal axes with respect to a sample fixed coordinate system. The crystal orientation may be specified by the three Eulerian angles $\varphi_{1} \phi \varphi_{2}$ and $d g$ is the orientation element.

$$
d g=\frac{\sin \phi}{8 \pi^{2}} d \varphi_{1} d \phi d \varphi_{2}
$$

With this definition, the volume of the whole orientation space is one. If a finite size $\Delta g$ of the orientation element is chosen then this determines the "resolving power" by which the function $f(g)$ is being represented. Many texture measurements are presently carried out with a step width of 5 degrees which corresponds (at $\phi=\pi / 2$ ) to an orientation element of $\Delta g=8.4 \times 10^{-6}$. In order to "scan" the whole orientation space with volume elements of this size, $N=1 / \Delta g \sim 118 \times 10^{3}$ volume elements and hence as many functional values are needed. If one takes crystal and sample symmetry into account, e.g. cubic crystal and orthorhombic sample symmetry (sheet symmetry) then the asymmetric unit of the orientation space is reduced by the symmetry factor $24 \times 4=96$. Hence, in this case $N=1230$ 
points are needed in order to represent the texture completely with a resolving power of 5 degrees.

The "physically necessary" resolving power may, however, be lower. It depends on the steepest slope of the function $f(g)$ which is to be represented. This property of a function can easily be estimated in the series representation

$$
f(g)=\sum_{\lambda=0}^{L} \sum_{\mu=1}^{M(\lambda)} \sum_{\nu=1}^{N(\lambda)} C_{\lambda}^{\mu v} \dot{T}_{\lambda}^{\mu \nu}(g) \geq 0
$$

The texture function is then represented by the coefficients $C_{\lambda}^{\mu v}$ which converge the faster the smaller the steepest slope of the function.

In order to estimate the convergence of the series Eq. (3), the mean absolute values of the coefficients $C_{\lambda}^{\mu \nu}$ may be plotted as a function of the degree $\lambda$. This is shown in Figure 1 for $90 \%$ cold rolled copper measured by neutron diffraction. The values are compared with the corresponding error quantities. It is seen that the coefficients converge to zero within the limit of error in the range $\lambda \leq 22$ which is thus often used as a standard value for the representation of an ODF.

The texture function is further restricted by the positivity condition also given in Eq. (3), which leads to an interrelation between the coefficients of even and odd order $\lambda$. The number of coefficients for some $\lambda$-values are given in Table 1 for the cubic orthorhombic case. If the number of even coefficients is considered as a lower limit and that of all coefficients as the upper limit, then between 124 and 185 values are needed in order to fully represent a texture function of this type.

Any method by which the function values $f(g)$ or the coefficients $C_{\lambda}^{\mu v}$ can be determined is a method of texture analysis. If all independent function values (as defined by the resolving power) or all coefficients with $\lambda \leq L$ are thus obtained, then we speak of complete texture analysis. If only some of the function values or of the coefficients are obtained then the method may be called partial texture analysis.

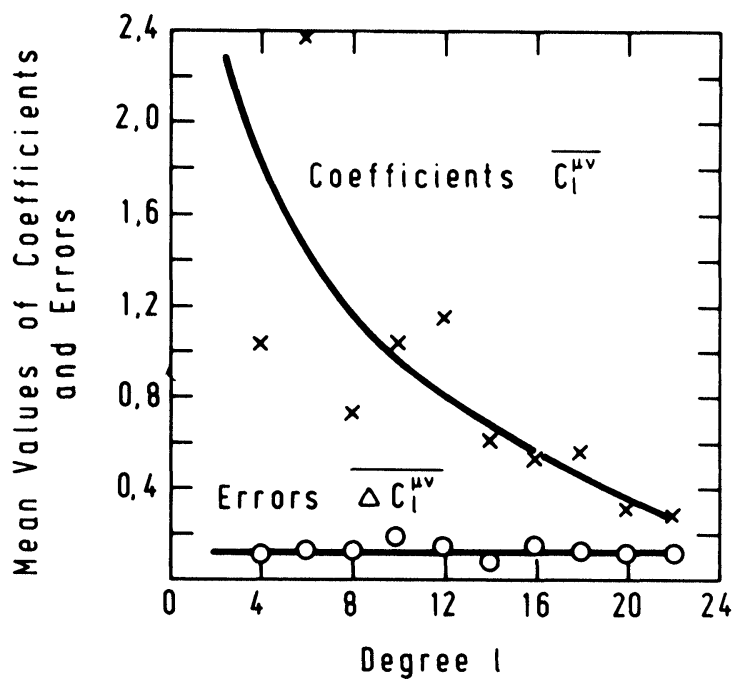

Figure 1 Convergence of the series of coefficients $C_{\lambda}^{\mu \nu}$. 
Table 1 Number of coefficients (cubic-orthorhombic)

\begin{tabular}{rrrrrrrr}
\hline$\lambda$ & $\mu$ & $v$ & $C_{\lambda}^{\mu \nu}$ & $\begin{array}{l}\Sigma C \\
\lambda \text { even }\end{array}$ & $\begin{array}{l}\Sigma C \\
\text { all } \lambda\end{array}$ & $F_{\lambda}^{\nu}$ & $\Sigma F$ \\
\hline 4 & 1 & 3 & 3 & 3 & 3 & 3 & 3 \\
9 & 1 & 5 & 5 & 12 & 17 & & \\
10 & 1 & 6 & 6 & 18 & 23 & 6 & 18 \\
12 & 2 & 7 & 14 & 32 & 37 & 7 & 25 \\
16 & 2 & 9 & 18 & 58 & 78 & 9 & 42 \\
22 & 2 & 12 & 24 & 124 & 185 & 12 & 75 \\
\hline \multicolumn{7}{c}{ lower upper } \\
\hline
\end{tabular}

It is the purpose of the present paper to compare various methods of partial texture analysis.

\section{METHODS OF TEXTURE ANALYSIS}

There are several different ways how to obtain information about the texture of a polycrystalline material. Some of these methods are principally capable of giving a complete texture analysis some others, however, can only give partial information, based on the underlying physical principles. Of course, also the "complete" methods may be used in a "reduced" form which gives only partial information.

\section{INDIVIDUAL ORIENTATION MEASUREMENTS}

The most straightforward way of texture determination is to measure the orientation $g(x y z)$ of small enough volume elements $\Delta V$ at all positions $x y z$ of the whole sample, Figure 2a. This can be done, for instance, by electron diffraction in the back-scattering mode at grid-points $x^{i} y^{j}$ in steps of $\Delta x, \Delta y$ over the whole sample surface, then removing the uppermost layer of the thickness $\Delta z$ and repeating the whole procedure until the whole sample volume has been measured.

If the material consists of well defined crystallites, it is sufficient to measure the orientations $g^{i}$ and volume fractions $v^{i}$ of all crystallites, Figure $2 \mathrm{~b}$. Both of these methods require of course, a vast amount of measurement. If we assume a grain size of $10 \mu \mathrm{m}$ and a sample size of $1 \mathrm{~mm}^{3}$ then $10^{6}$ crystal orientations $g^{i}$ and volume fractions $v^{i}$ must be measured in the second variant. In the first variant a step width of, say, $1 \mu \mathrm{m}$ is necessary which requires $10^{8}$ measurements.

The texture function can be represented by the coefficients $C_{\lambda}^{\mu \nu}$ which are expressed by the measured orientations in the forms

$$
\begin{gathered}
C_{\lambda}^{\mu v}=(2 \lambda+1) \frac{\Delta v}{v} \sum_{i, j, k} \dot{T}_{\lambda}^{\mu v}\left(g\left(x_{i}, y_{i}, z_{k}\right)\right) \\
C_{\lambda}^{\mu v}=(2 \lambda+1) \frac{1}{v} \sum_{i} v_{i} \dot{T}_{\lambda}^{\mu v}\left(g_{i}\right)
\end{gathered}
$$



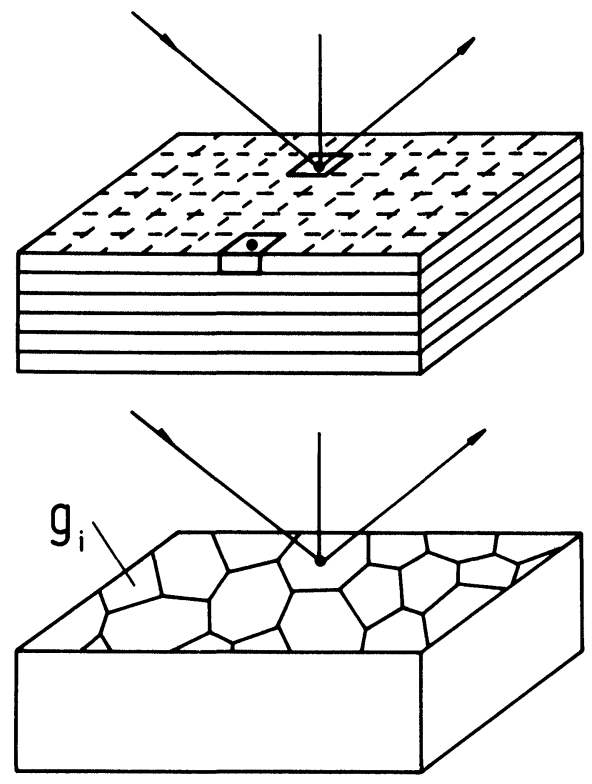

(a)

(b)

Figure 2 Individual orientation measurement. (a) In equidistant volume elements; (b) in individual grains.

for the first and second variant respectively. This is the most straightforward way of texture determination based directly on the definition Eq. (1). It is, of course, also the most laborious way.

The method may be simplified by assuming a statistically homogeneous sample. Then a two-dimensional section may be considered as being representative.

Single orientation measurement thus provides the complete texture informations. It must, however, be mentioned that the formulae Eqs. $(4,5)$ are correct only if a statistically representative set of crystals $i$ or volume elements (ijk) is being used which may often not be the case.

\section{POLE FIGURE INVERSION}

The most frequently used way of texture determination is that of a pole figure measurement followed by pole figure inversion. A pole figure is the orientation distribution of the sample volume of which a specific crystal direction $h$ is parallel to a specific sample direction $y$

$$
P_{h}(y)=\frac{d V / V}{d y} ; \quad y=\{\alpha, \beta\}
$$

Thereby $d y$ is the solid angular element

$$
d y=\sin \alpha d \alpha d \beta
$$

With this definition the area of the whole pole sphere is $4 \pi$. If we use a finite angular element $\Delta y$ of, say, 5 degrees in $\alpha$ and $\beta$ (at $\alpha=\pi / 2$ ) then it is $\Delta y / 4 \pi=6 \times 10^{-4}$. This means that $N=1650$ solid angular elements and hence as 
many function values are needed in order to represent a pole figure. This number may be reduced by the sample symmetry. In the case of orthorhombic symmetry only one octant of the pole figure is symmetrically independent. Hence, $N=206$ values are sufficient to describe a pole figure with this resolving power (equal area scan).

A pole figure can be expressed in terms of a series expansion

$$
P_{h}(y)=\sum_{\lambda=0}^{L} \sum_{v=1}^{N(\lambda)} F_{\lambda}^{v}(h) \cdot \dot{k}_{\lambda}^{v}(y)
$$

The coefficients $F_{\lambda}^{v}(h)$ are obtained from the measured pole figure values by the inversion of Eq. (8)

$$
F_{\lambda}^{v}(h)=\oint P_{h}(y) \cdot \dot{k}_{\lambda}^{* v}(y) d y
$$

Again, the "physically necessary" resolving power can be estimated by the convergence of the series Eq. (8) which is similar to that of the coefficients $C_{\lambda}^{\mu \nu}$ estimated in Figure 1. The number of coefficients $F_{\lambda}^{v}$ of various degrees $\lambda$ is also given in Table 1 for the orthorhombic case. (Because of the centrosymmetry of experimentally determined pole figures only the coefficients of even order $\lambda$ are different from zero). Hence, an orthorhombic pole figure is represented completely by $N=75$ coefficients (in an approximation up to $\lambda \leq 22$ ). A pole figure is an integral over the texture function

$$
P_{h}(y)=\frac{1}{2 \pi} \int_{h \| y} f(g) d \psi
$$

Hence, the coefficients $F_{\lambda}^{v}$ are related to the coefficients $C_{\lambda}^{\mu v}$ by the expression

$$
F_{\lambda}^{v}(h)=\frac{4 \pi}{2 \lambda+1} \sum_{\mu=1}^{M(\lambda)} C_{\lambda}^{\mu \nu} \cdot \dot{k}_{\lambda}^{* \mu}(h)
$$

Pole figure measurement has to be followed by "pole figure inversion," i.e. the determination of the coefficients $C_{\lambda}^{\mu v}$ by solving Eq. (11) with the $F_{\lambda}^{v}(h)$ of several pole figures $h$ given. In Eq. (11) $M(\lambda)$ pole figures are needed for a unique solution. The functions $M(\lambda)$ depends on the crystal symmetry (see e.g. Bunge 1982). As an example in the cubic case it is $M=2$ up to $\lambda=22$. Hence, in this case, two pole figures with $2 \times 75=150$ coefficients $F_{\lambda}^{v}$ are sufficient to determine the 124 even coefficients $C_{\lambda}^{\mu \nu}$ up to $\lambda=22$. The 61 odd coefficients are not strictly fixed by the even coefficients. In most cases they are, however, approximately fixed by the even ones. In this sense pole figure measurement followed by pole figure inversion is a method which gives the complete information about the texture function $f(g)$. Strictly speaking, however, there is some degree of freedom in the determination of the odd coefficients. Then the ODF can only be obtained with an "uncertainty margin".

\section{INCOMPLETE POLE FIGURES}

In order to obtain the coefficients $F_{\lambda}^{\nu}(h)$ by Eq. (9) the pole figures must have been measured in the whole range of sample directions $y$ (or the asymmetric unit, 
e.g. one octant of the pole sphere). In many cases this is not practicable. Then pole figures are being measured only in an incomplete range e.g. for

$$
0 \leq \alpha \leq \alpha_{\max }<90^{\circ}
$$

This means according to Eq. (10) that crystals in the range $\alpha_{\max }<\alpha \leq 90^{\circ}$ are excluded from the measurement, which is thus incomplete. On the other hand, if several incomplete pole figures are being used, then information about the missing part of one pole figure is implicitly contained in the measured parts of other pole figures. Furthermore, in highly symmetric crystal symmetries, the incomplete range of even one pole figure contains contributions of at least one of several symmetrically equivalent directions $\{h\}$ of all crystallites. By a slight modification of the above-mentioned method, it is thus possible to obtain the complete texture information also from incomplete pole figure measurements. Hence, also the "incomplete pole figure method" is a method of "complete texture analysis."

Pole figure measurements are mostly carried out by X-ray diffraction. Similar methods are, however, also available with neutron and electron diffraction.

\section{FIXED ANGLE TEXTURE ANALYZER}

Texture determination has also been carried out by a "fixed angle texture analyzer" (Kopineck 1986, Kopineck and Otten 1987, Kopineck and Bunge 1988). In this method an incident white X-ray beam is diffracted in the sample. Five energy dispersive detectors are arranged in five angular positions in transmission geometry, Figure 3a. Each detector measures five diffraction peaks. Hence, this method gives five pole figure values near to the equator of each of five pole figures as is shown schematically in Figure $3 \mathrm{~b}$. Thus, 25 pole density values are obtained. According to Eq. (10), each pole density value corresponds to a group of crystallites which have their crystal direction $h$ parallel to the sample direction $y$. All other crystallites which do not have one of these specific orientations are not "seen" by the method. Hence, the fixed angle texture analyzer can only give partial information about the texture. This is also seen if one compares the number of 25 pole density values thus obtained with the necessary number of 124 coefficients needed in order to represent a texture with a series approximation of $L=22$.

In order to evaluate the experimental data obtained by the fixed angle texture analyzer, a low-order model texture is assumed e.g. with $L=4$, the coefficients of which are then calculated by a least squares deviation assumption with respect to the measured pole density values. Combining Eqs. (8) and (11) the pole densities of the correct texture and of the (fourth-order) model texture can be written

$$
\begin{aligned}
P_{h}(y) & =\sum_{\lambda=0}^{L} \sum_{\mu=1}^{M(\lambda)} \sum_{v=1}^{N(\lambda)} \frac{4 \pi}{2 \lambda+1} C_{\lambda}^{\mu v} k_{\lambda}^{* \mu}(h) \dot{k}_{\lambda}^{v}(y) \\
{ }^{M} P_{h}(y) & =\sum_{v=1}^{3} \frac{4 \pi}{9}{ }^{M} C_{4}^{1 v}{ }^{*}{ }_{4}^{* 1}(h) \dot{k}_{4}^{v}(y)
\end{aligned}
$$

(thereby the cubic orthorhombic case and $L_{0}=4$ was assumed but other cases are 


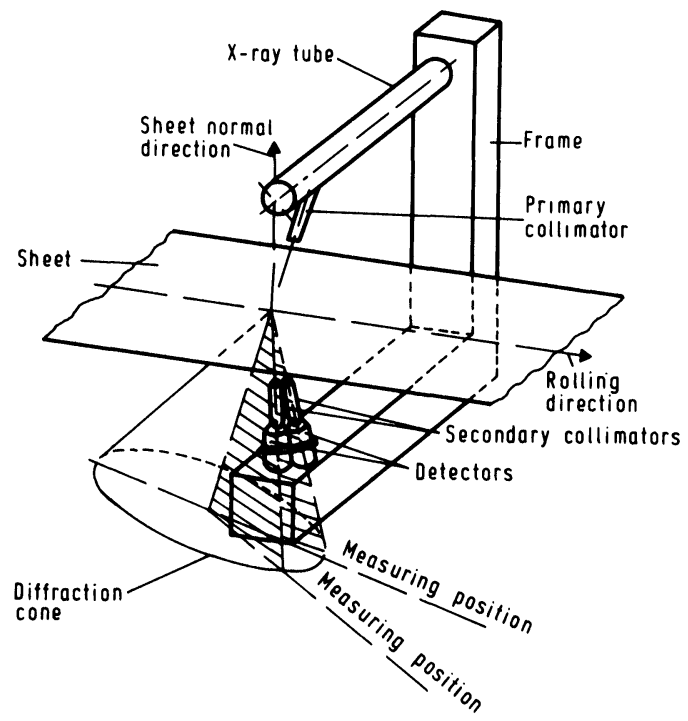

(a)

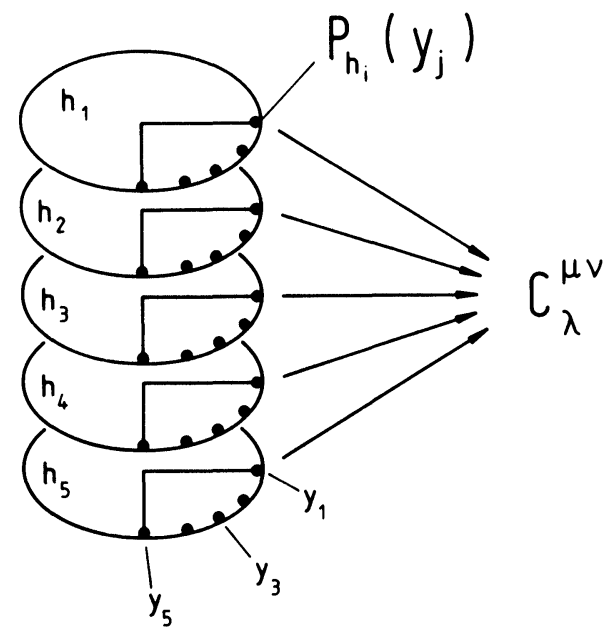

(b)

Figure 3 Fixed angle texture analyzer. (a) Principle design; (b) position of the pole figure points.

also possible). The coefficients ${ }^{M} C_{4}^{1 v}$ are obtained according to the condition

$$
\sum_{i=1}^{i_{\max }} \sum_{j=1}^{j_{\max }}\left[{ }^{M} P_{h_{i}}\left(y_{j}\right)-P_{h_{i}}\left(y_{j}\right)\right]^{2}=\operatorname{Min}
$$

Hence, a fourth order texture function is determined in such a way that the real pole figure values in 25 selected points are represented with the smallest possible 
deviation by the model texture. The texture coefficients ${ }^{M} C_{4}^{1 v}$ can then be expressed in the form

$$
{ }^{M} C_{4}^{1 v}=\sum_{i=1}^{i_{\max }} \sum_{j=1}^{j_{\max }} a_{4}^{1 v}(i, j)\left[P_{h_{i}}\left(y_{i}\right)-1\right]
$$

where $a_{4}^{1 v}$ are numerical coefficients which depend on the selection of all $h_{i}$ - and $y_{j}$-values (Bunge and Wang, 1987). The so obtained coefficients ${ }^{M} C_{4}^{1 v}$ deviate from those of the real texture but they would converge towards the real texture coefficients if the number of points $i, j$ were increased much beyond the chosen 25.

$$
\begin{aligned}
& { }^{M} C_{4}^{1 v} \neq C_{4}^{1 v} \\
& { }^{M} C_{4}^{1 v} \rightarrow C_{4}^{1 v} \text { for } i, j \rightarrow \infty
\end{aligned}
$$

The deviation of the model coefficients ${ }^{M} C_{4}^{1 v}$ from the true ones is due to the fact, that the measured pole figure values, Eq. (13) also contain higher order coefficients of the real texture which are not taken into account in Eq. (14).

In this sense, texture analysis by a fixed angle texture analyzer is a method of partial texture analysis (lower order coefficients only) and it is approximative in the coefficients with respect to the true coefficients.

\section{TEXTURE DETERMINATION BY PHYSICAL METHODS}

A partial texture analysis of principally different character is obtained by evaluating the macroscopic anisotropy of any physical property which is anisotropic in single crystals, Figure 4. Among these properties, the magnetic anisotropy has obtained the widest interest since it is easiest to measure. Magnetic texture analyzers have been developed based on this principle (see e.g. Wassermann and Grewen, 1962). But also elastic properties are coming more and more into use, presently. In principle the method can be applied to any anisotropic property of which the simple, texture-weighted mean value can be measured in a sufficient number of directions. A certain difficulty may, however, arise according to the nature of the mean value as will be considered later.

The direction dependence of a physical property with respect to the crystal axes may be expressed by a function $E(h)$ which can be developed into a series of

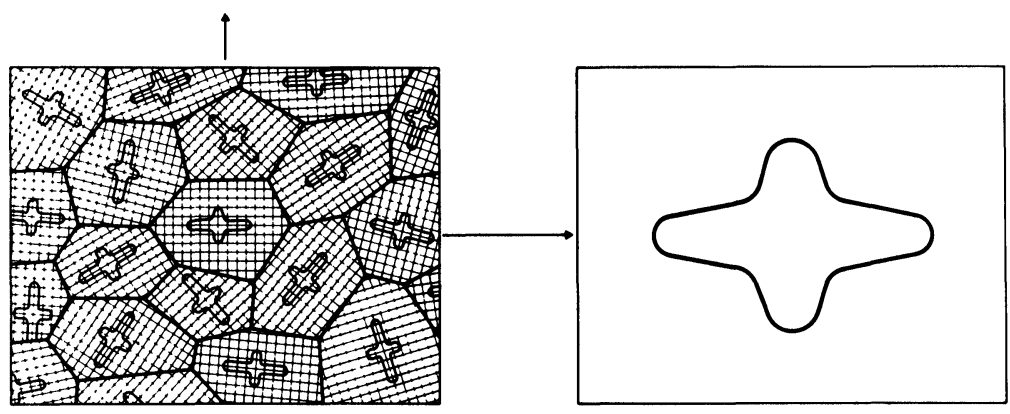

Figure 4 Orientation mean value of an anisotropic physical property. 
spherical surface harmonics $\dot{k}_{\lambda}^{\mu}$ with the crystal symmetry, depending on the crystal direction $h$ (see e.g. Bunge 1969, 1982)

$$
E(h)=\sum_{\lambda=0}^{L_{0}} \sum_{\mu=1}^{M(\lambda)} e_{\lambda}^{\mu} \cdot \dot{k}_{\lambda}^{\mu}(h)
$$

For most of all physical properties Eq. (18) is a finite series with a low value of $L_{0}$ as is shown in Table 2. In some other cases (such as the plastic properties for instance) the series converges very rapidly with increasing $\lambda$ such that a low $L_{0}$-value represents a very good approximation. It is assumed in Eq. (18) that the property constants $e_{\lambda}^{\mu}$ are known (e.g. from property data files). Thereby $E(h)$ is often expressed in terms of other functions than the surface harmonics and hence with other constants than $e_{\lambda}^{\mu}$. In these cases a transformation from the tabulated property constants to $e_{\lambda}^{\mu}$ is, however, easily possible (see e.g. Bunge, 1969, 1982).

The direction dependence of the corresponding property in a polycrystalline material can be expressed similar to Eq. (18) in the form

$$
\bar{E}(y)=\sum_{\lambda=0}^{L_{0}} \sum_{v=1}^{N(\lambda)} \bar{e}_{\lambda}^{v} \cdot \dot{k}_{\lambda}^{v}(y)
$$

where $\dot{k}_{\lambda}^{v}$ are surface harmonics with the sample symmetry, and $y$ is the sample direction. Thereby $L_{0}$ is the same value as in Eq. (18), i.e. generally a low value.

If property measurements are to be used for texture determination then the coefficients $\bar{e}_{\lambda}^{v}$ must be calculated from $\bar{E}(y)$ values corresponding to different sample direction $y$. Using the orthogonality properties of surface harmonics,

Table 2 Order $L_{0}$ of the anisotropy of physical properties

\begin{tabular}{ll}
\hline Property & Order $L_{0}$ \\
\hline $\begin{array}{l}\text { Tensor properties } \\
\text { (rank } n \text { ) }\end{array}$ & $n$ \\
$\begin{array}{l}\text { rank } 2 \\
\text { electric conductivity } \\
\text { dielectric susceptibility } \\
\text { optic refraction index } \\
\text { thermal expansion } \\
\text { diffusion coefficient } \\
\text { rank } 3\end{array}$ & 2 \\
$\quad$ piezoelectric effect \\
rank 4 \\
$\quad \begin{array}{l}\text { elasticity } \\
\text { magnetostriction } \\
\text { electrostriction } \\
\text { induced magnetic anisotropy }\end{array}$ \\
$\begin{array}{l}\text { Magnetization energy } \\
\text { (core losses) }\end{array}$ \\
$\begin{array}{l}\text { Plastic anisotropy } \\
\quad \text {-value } \\
\text { yield locus } \\
\text { earing }\end{array}$ \\
\hline
\end{tabular}


Eq. (19) can be inverted by an integral expression

$$
\bar{e}_{\lambda}^{v}=\oint \bar{E}(y) \cdot \dot{k}_{\lambda}^{* v}(y) d y
$$

This requires, however, the measurement of the property $\bar{E}(y)$ as a continuous function of the sample direction $y$. Because of the low order nature of Eq. (19) the coefficients $\bar{e}_{\lambda}^{v}$ can also be obtained from a finite number of property measurements taken in the sample directions $y_{i}$. Then Eq. (19) assumes the form of a system of linear equations

$$
\bar{E}\left(y_{i}\right)=\sum_{\lambda=0}^{L_{0}} \sum_{v=1}^{N(\lambda)} \bar{e}_{\lambda}^{v} \cdot k_{\lambda}^{v}\left(y_{i}\right) ; \quad i=1 \cdots I
$$

which can be solved for the $\bar{e}_{\lambda \text {. }}^{v}$.

As will be seen later, an important case is the one of cubic crystal symmetry and orthorhombic sample symmetry and $L_{0}=4$. Then Eq. (21) takes on the simple form

$$
\bar{E}\left(y_{i}\right)=\bar{e}_{0}^{1} k_{0}^{1}+\sum_{v=1}^{3} \bar{e}_{4}^{v} \cdot \dot{k}_{4}^{v}\left(y_{i}\right) ; \quad i=1 \cdots I
$$

Thereby the first term is direction independent, it corresponds to a material with random crystal orientation, and the three terms in the sum describe the anisotropy of the polycrystal property in a textured material. Since Eq. (22) contains only three unknown quantities $\bar{e}_{\lambda}^{v}$, it can be solved with $I=3$ measured property values $E\left(y_{i}\right)$ taken, for instance, in rolling, transverse, and normal direction of a sheet material

$$
\begin{aligned}
& y_{1}=\left\{\alpha=90^{\circ}, \beta=0^{\circ}\right\}=R D \\
& y_{2}=\left\{\alpha=90^{\circ}, \beta=90^{\circ}\right\}=T D \\
& y_{3}=\left\{\alpha=0^{\circ}, \beta=0^{\circ}\right\}=N D
\end{aligned}
$$

If the properties of the individual crystallites are not modified by interaction with their neighbours in the polycrystalline material then the polycrystal property will be the simple, texture weighted mean value of the single crystal properties of all crystallites as is shown in Figure 4 . In this case the macroscopic property $\bar{E}(y)$ is expressed by the microscopic property $E(h)$ and the texture in the form

$$
\bar{E}(y)=\frac{1}{4 \pi} \oint E(h) \cdot A(h, y) d h
$$

where $A(h, y)$ is identical with the pole figure, Eq. (13) with the only difference, that now the crystal direction $h$ is allowed to vary continuously whereas in Eq. (13) it was assumed to be fixed. Hence, $A(h, y)$ is expressed by the texture coefficients

$$
A(h, y)=\sum_{\lambda=0}^{L} \sum_{\mu=1}^{M(\lambda)} \sum_{\nu=1}^{N(\lambda)} \frac{4 \pi}{2 \lambda+1} C_{\lambda}^{\mu v} \dot{k}_{\lambda}^{* \mu}(h) \dot{k}_{\lambda}^{v}(y)
$$


With Eq. (19) and Eq. (25), Eq. (24) can be expressed in terms of the coefficients $C_{\lambda}^{\mu v}$

$$
\bar{e}_{\lambda}^{\nu}=\sum_{\mu=1}^{M(\lambda)} \frac{e_{\lambda}^{\mu} \cdot C_{\lambda}^{\mu \nu}}{2 \lambda+1} ; \quad \lambda \leq L_{0}
$$

Since the anisotropy constants $e_{\lambda}^{\mu}$ are zero for $\lambda>L_{0}$, the texture coefficients $C_{\lambda}^{\mu v}$ for $\lambda>L_{0}$ do not enter the mean value expression Eq. (26) which is equivalent with Eq. (24). The physical property of a polycrystalline material is completely independent of these texture coefficients. If a texture would contain only coefficients $C_{\lambda}^{\mu \nu}$ with $\lambda>L_{0}$ then its physical property $\bar{E}(y)$ would be completely isotropic. Higher order texture coefficients $C_{\lambda}^{\mu \nu}$ with $\lambda>L_{0}$ cannot be "seen" by a physical property with $\lambda \leq L_{0}$. Hence, physical property measurements can only be methods of "partial texture analysis", the "resolving power" being $L_{0}$.

It is seen that $\lambda \leq L_{0}$ is a necessary but not yet sufficient condition for the solution of Eq. (26) for the texture coefficients $C_{\lambda}^{\mu \nu}$. A second condition is

$$
M(\lambda)=1
$$

which is fulfilled for $\lambda \leq \lambda_{\max }$ depending on the crystal symmetry as is shown in Table 3. In this case Eq. (26) becomes

$$
\bar{e}_{\lambda}^{v}=\frac{e_{\lambda}^{1} \cdot C_{\lambda}^{1 v}}{2 \lambda+1} ; \quad \lambda \leq \lambda_{\max }
$$

Equation (28) can be uniquely solved for the texture coefficients $C_{\lambda}^{1 v}$ if the single crystal constants $e_{\lambda}^{1}$ are known and the polycrystal constants $\bar{e}_{\lambda}^{v}$ have been measured. Hence, physical property measurements can give information on the texture coefficients $C_{\lambda}^{1 v}$ for $\lambda \leq\left[L_{0}, \lambda_{\max }\right]$. These coefficients are generally not sufficient to calculate the orientation density $f(g)$ for a given crystal orientation $g$ in the sample, Eq. (3) or the pole density $P_{h}(y)$ of a given pole figure $h$ in the sample direction $y$, Eq. (8). The values $C_{\lambda}^{1 v}$ obtained from physical property measurements are, however, the correct values of the true texture and not values of an approximative model texture as was the case with the fixed angle texture analyzer. This difference is due to the fact that in Eq. (24) all crystals of the sample contribute to each property value $\bar{E}(y)$ in any sample direction $y$ whereas in Eq. (13) only a specific group of crystals, i.e. those defined in Eq. (10), contribute to the experimental values $P_{h i}\left(y_{j}\right)$ on which this method was based.

Hence, physical property measurements and the fixed angle texture analyzer both provide methods of partial texture analysis, they are, however, complementary to each other in as far as the fixed angle texture analyzer gives correct pole figure values and approximative $C$-coefficients whereas the physical methods give

Table 3 Maximum degree $\lambda_{\max }$ for $M(\lambda)=1$

\begin{tabular}{ll}
\hline Symmetry & Degree $\lambda_{\max }$ \\
\hline Cubic & 10 \\
Hexagonal & 4 \\
Tetragonal & 2 \\
Orthorhombic & 1 \\
\hline
\end{tabular}


Table 4 Characteristics of methods of partial texture analysis

\begin{tabular}{|l|ll|}
\hline Method & $\begin{array}{l}\text { Fixed angle } \\
\text { texture analyzer }\end{array}$ & $\begin{array}{l}\text { Physical property } \\
\text { measurements }\end{array}$ \\
\hline $\begin{array}{l}\text { Pole density } \\
c \text {-coefficients }\end{array}$ & $\begin{array}{l}\text { Correct } \\
\text { Approximative }\end{array}$ & $\begin{array}{l}\text { Approximative } \\
\text { Correct }\end{array}$ \\
\hline
\end{tabular}

correct $C$-coefficients but by approximative pole figure values as is shown in Table 4.

The determination of texture coefficients $C_{4}^{1 v}$ according to Eq. (28) is based on two assumptions which are, however, not always fulfilled. These are:

(1) The orientation dependence of the single crystal property according to Eq.

(18) must be known.

(2) The mean value expression Eq. (24) must be valid.

Both conditions are quite well fulfilled, for instance, in the case of the magnetization energy of ferromagnetic crystals in the vincinity of saturation. The orientation dependence of this energy can be described in the cubic or hexagonal case in the forms

$$
\begin{aligned}
& E(h)=K_{1}\left(h_{1}^{2} h_{2}^{2}+h_{2}^{2} h_{3}^{2}+h_{3}^{2} h_{1}^{2}\right)+K_{1} h_{1}^{2} h_{2}^{2} h_{3}^{2} \\
& E(h)=K_{1}^{\prime} \cos ^{2} \theta+K_{2}^{\prime} \cos ^{4} \theta
\end{aligned}
$$

The constants $K_{1}, K_{2}$ and $K_{1}^{\prime}, K_{2}^{\prime}$ can be transformed into the corresponding $e_{\lambda}^{\mu}$-values of Eq. (18) (see e.g. Bunge 1969, 1982). Furthermore, in the range of saturation, the magnetization vector is nearly parallel in all crystallites of a polycrystalline sample. Hence, there is only very little influence of neighbouring crystals on the magnetization energy of a considered crystal. The magnetization energy of the polycrystal is then the mean value of the magnetization energies of the crystallites of the materials as assumed in Eq. (24). The direction dependence of the magnetization energy, Eq. (19), can be deduced from torque curve measurements. Hence, torque curve measurements in saturation (see e.g. Cullity 1972) can be used to measure the texture coefficients $C_{\lambda}^{1 v}$ (up to $\lambda=6$ in the cubic case).

The magnetic properties in other parts of the hysteresis loop cannot be expressed by a unique function of crystal orientation. In a single crystal the distribution of the magnetization directions then generally depends on the magnetic history of the material and not only on crystal orientation. Furthermore, there will also be interactions across the grain boundaries by differently oriented domains. Hence, in this case neither condition (1) nor condition (2) are strictly fulfilled. Magnetic measurements including non-saturation states (i.e. all alternating current measurements) are thus not well suited to determine texture coefficients. Nevertheless, it is often possible to calibrate such measurements empirically. They can then also be used-at least qualitatively-to obtain information on low-order texture coefficients. Magnetic measurements based on hysteresis losses, eddy current, permeability, coercivity or Barkhausen noise fall within this category. They provide very handy methods for "texture inspection" and they may be calibrated empirically in fortunate cases to obtain low order texture coefficients. It is, however, difficult in these cases to formulate strict 
relationships between the measured property values and the texture coefficients without taking recourse to empirical "fitting parameters."

Young's modulus measurements (or more generally the elastic constants) are another example of an anisotropic property which has been used for texture inspection or partial texture measurement. In this case the orientation dependence in single crystals can be expressed by the fourth-rank tensor components in the anisotropic Hooke's law in one of two forms

with

$$
\begin{aligned}
\quad \sigma_{i j}=C_{i j k l} \cdot \varepsilon_{k l} \\
\varepsilon_{i j}=S_{i j k l} \cdot \sigma_{k l}
\end{aligned}
$$

$$
C_{i j k l}=\left[S_{i j k l}\right]^{-1}
$$

In a polycrystal, the continuity of the strains $\varepsilon$ and the equilibrium of the stresses $\sigma$ across the grain boundaries must be fulfilled. This means an interaction between neighbouring crystals which depends not only on the orientation of the considered crystal but also on the orientations of its neighbours. Hence, the orientation mean value expression Eq. (24) is not strictly valid.

If one assumes constant strains $\varepsilon_{k l}$ in all crystals (Voigt, 1928) or constant stresses $\sigma_{k l}$ (Reuss, 1929) as upper and lower bound assumptions then two different mean values of the single crystal properties are obtained

$$
\begin{aligned}
\bar{C}_{i j k l}^{V} & =\oint C_{i j k l}(g) \cdot f(g) d g \\
\bar{S}_{i j k l}^{R} & =\oint S_{i j k l}(g) \cdot f(g) d g
\end{aligned}
$$

where $C_{i j k l}(g)$ and $S_{i j k l}(g)$ are the tensor components referred to the sample coordinate system. In contrast to Eq. (33) it is

$$
\bar{S}_{i j k l}^{R} \neq \bar{S}_{i j k l}^{V}=\left[\bar{C}_{i j k l}^{V}\right]^{-1}
$$

In the case of uniaxial tension the Voigt approximation is correct in a polycrystalline structure consisting of pencil-shaped crystals, whereas the Reuss approximation holds for plate-like crystals. An example of calculated anisotropies with the same texture and the two assumptions is given in Figure 5 for a copper sheet. The difference between the two approximations increases with increasing elastic anisotropy (see e.g. Chung and Buessem 1967). The true mean value may be somewhere between the two assumptions. As an approximation, a weighted mean value between the Voigt and Reuss assumption may be taken

$$
\tilde{S}_{i j k l}=m \cdot \bar{S}_{i j k l}^{V}+(m-1) \cdot \bar{S}_{i j k l}^{R}
$$

where $m$ is a certain "grain shape factor" which varies between one and zero for shapes between "pencil" and "plate." For the case of nearly equiaxed grains, Hill (1952) assumed $m=0.5$. It is found experimentally that Eq. (37) with $m=0.5$ is a quite satisfactory approximation from the practical point of view (though not from a theoretical one, see e.g. Kröner 1958). 


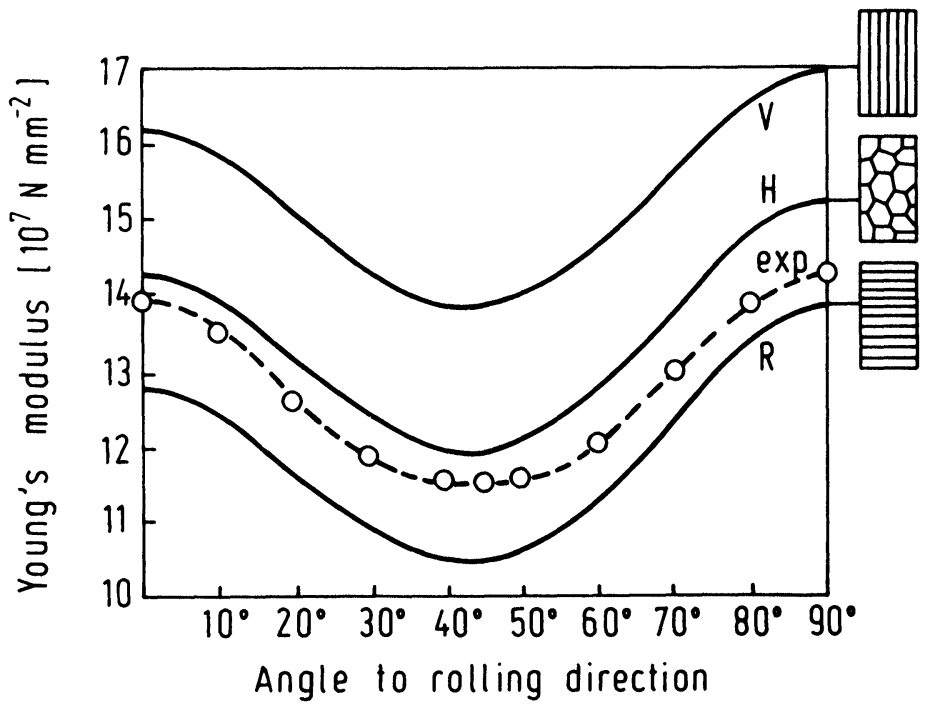

Figure 5 Young's modulus in a copper sheet according to the Voigt, Reuss, Hill approximations calculated from texture data and experimental values.

The tensor components $C_{i j k l}(g)$ and $S_{i j k l}(g)$ in Eqs. (34), (35) are fourth order functions of the crystal orientation $g$. Hence, the mean values $\bar{C}_{i j k l}^{V}$ and $\bar{S}_{i j k l}^{R}$ can be expressed in terms of the texture coefficients $C_{\lambda}^{\mu \nu}$ up to $L_{0}=4$. In the case of cubic orthorhombic symmetry this reads (see e.g. Bunge 1968, 1968, 1982)

$$
\begin{aligned}
& \bar{C}_{i j k l}^{V}=C_{i j k l}^{0}+\left(C_{1111}^{0}-C_{1122}^{0}-2 C_{1212}^{0}\right) \cdot A_{i j k l} \\
& \bar{S}_{i j k l}^{V}=S_{i j k l}^{0}+\left(S_{1111}^{0}-S_{1122}^{0}-2 S_{1212}^{0}\right) \cdot A_{i j k l}
\end{aligned}
$$

where $C_{i j k l}^{0}$ and $S_{i j k l}^{0}$ are the corresponding tensor components referred to the

Table 5 Averaging factors for the elastic tensor (cubic-

\begin{tabular}{|c|c|c|c|c|}
\hline \multirow[t]{2}{*}{$i j k l$} & \multirow{2}{*}{$\begin{array}{l}\text { Random } \\
\bar{a}_{i j k l}\end{array}$} & \multicolumn{3}{|c|}{ Texture } \\
\hline & & $a_{i j k l}^{1}$ & $a_{i j k l}^{2}$ & $a_{i j k l}^{3}$ \\
\hline 1111 & -0.4 & +0.021818 & -0.032530 & +0.043032 \\
\hline 2222 & -0.4 & +0.021818 & +0.032530 & +0.043032 \\
\hline 3333 & -0.4 & +0.058182 & 0 & 0 \\
\hline 1122 & +0.2 & +0.007273 & 0 & -0.043032 \\
\hline 1133 & +0.2 & -0.029091 & +0.032530 & 0 \\
\hline 2233 & +0.2 & -0.029091 & -0.032530 & 0 \\
\hline 1212 & +0.2 & +0.007273 & 0 & -0.043032 \\
\hline 1313 & +0.2 & -0.029091 & +0.032530 & 0 \\
\hline 2323 & +0.2 & -0.029091 & -0.032530 & 0 \\
\hline
\end{tabular}
orthorhombic case) 
crystal axes. The quantities $A_{i j k l}$ depend on the texture coefficients

$$
A_{i j k l}=\bar{a}_{i j k l}+a_{i j k l}^{1} \cdot C_{4}^{11}+a_{i j k l}^{2} \cdot C_{4}^{12}+a_{i j k l}^{3} \cdot C_{4}^{13}
$$

The purely mathematical constants $a_{i j k l}^{v}$ are tabulated in Table 5 (see Bunge 1968, for lower symmetries see e.g. Morris 1969). The quantities $\bar{S}_{i j k l}^{R}$ (and with Eq. (36) also $\left.\bar{C}_{i j k l}^{V}\right)$ are related to the Young's modulus in various sample directions $y=\left\{y_{1} y_{2} y_{3}\right\}$

$$
\begin{aligned}
\bar{E}(y)^{-1}= & y_{1}^{4} \tilde{S}_{1111}+y_{2}^{4} \tilde{S}_{2222}+y_{3}^{4} \tilde{S}_{3333}+2 y_{1}^{2} y_{2}^{2}\left(\tilde{S}_{1122}+2 \tilde{S}_{1212}\right) \\
& +2 y_{1}^{2} y_{3}^{2}\left(\tilde{S}_{1133}+2 \tilde{S}_{1313}\right)+2 y_{2}^{2} y_{3}^{2}\left(\tilde{S}_{2233}+2 \tilde{S}_{2323}\right)
\end{aligned}
$$

The coefficients $C_{4}^{1 v}$ can be calculated by solving Eq. (40) when at least three $A_{i j k l}$ are known. These can be obtained from Eqs. (38), (39) and (41) from at least three Young's modulus values measured in at least three independent sample directions $y$. These directions may be chosen, for instance, as the three main directions (e.g. normal direction, transverse direction and rolling direction of a sheet, Eq. (23)). With the Young's moduli measured in these three directions one obtains from Eqs. (39), (40), (41) in the Reuss approximation

$$
\begin{aligned}
& \frac{\bar{E}_{R D}^{-1}-S_{1111}^{0}}{S^{a}}-\bar{a}_{1111}=a_{1111}^{1} \cdot C_{4}^{11}+a_{1111}^{2} \cdot C_{4}^{12}+a_{1111}^{3} \cdot C_{4}^{13} \\
& \frac{\bar{E}_{T D}^{-1}-S_{1111}^{0}}{S^{a}}-\bar{a}_{1111}=a_{2222}^{1} \cdot C_{4}^{11}+a_{2222}^{2} \cdot C_{4}^{12}+a_{2222}^{3} \cdot C_{4}^{13} \\
& \frac{\bar{E}_{N D}^{-1}-S_{1111}^{0}}{S^{a}}-\bar{a}_{1111}=a_{3333}^{1} \cdot C_{4}^{11}+a_{3333}^{2} \cdot C_{4}^{12}+a_{3333}^{3} \cdot C_{4}^{13}
\end{aligned}
$$

where $S^{a}$ is the term in brackets in Eq. (38) i.e. the absolute value of the elastic anisotropy. Equation (42) can be solved for the three texture coefficients $C_{4}^{1 v}$. In the Voigt approximation, the relationship is a bit more complicated. A good approximation is, however, obtained in this case with a similar formula to Eq. (40) with $\bar{a}_{i j k l}=\tilde{a}_{i j k l}^{R}$ replaced by $\bar{a}_{i j k l}^{V}$.

Hence, although in the case of elastic properties a simple mean value relationship Eq. (24) does not hold theoretically, the practical mean value Eq. (37) also leads to a relationship containing only texture coefficients up to fourth order. In the case of cubic-orthorhombic symmetry these are the three coefficients $C_{4}^{1 v}$ which can thus be determined from Young's modulus measurements. Higher order coefficients do not enter the expressions. They can thus not be obtained from Young's modulus measurements in the way considered above. (See, however, Bunge 1974 and Sakata, Daniel and Jonas 1989)

\section{CONCLUSIONS}

Complete texture determination requires experimental methods which incorporate all crystals of the sample and which are highly "discriminative" with respect to 
the sample directions. Two different methods can thus be distinguished which fulfill these requirements:

(1) Single orientation measurements of all crystals of the sample (or of at least a representative number of crystals)

(2) Pole figure measurements (as a continuous function of the sample direction)

The result of complete texture analysis can be expressed either by the orientation distribution function (ODF), by several pole figures or by a complete set of texture coefficients $C_{\lambda}^{\mu \nu}$.

Partial texture analysis does not give the full information about the texture. Two different groups of partial texture measurements may be distinguished which are somehow complementary to each other. These are:

(1) The fixed angle texture analyzer, which gives a limited number of correct pole density values and approximated $C$-coefficients.

(2) Anisotropy measurements of physical properties such as magnetic anisotropy, Young's modulus, and others which give a limited number of correct $C$-coefficients but only approximated pole density or orientation density values.

\section{References}

Bunge, H. J. (1968). Über die elastischen Konstanten kubischer Materialien mit beliebiger Textur. Kristall u. Technik 3, 431-438.

Bunge, H. J. (1969). Mathematische Methoden der Texturanalyse. Akademie Verlag Berlin.

Bunge, H. J. (1974). The Effective Elastic Constants of Textured Polycrystals in Second Order Approximation. Kristall Techn. 9, 413-423.

Bunge, H. J. (1982). Texture Analysis in Materials Science. Butterworths Publ. London.

Bunge, H. J., Wang, F. (1987). Computational Problems in Low-Resolution Texture Analysis. In: Theoretical Methods of Texture Analysis. Ed. H. J. Bunge. DGM Informationsgesellschaft, Oberursel, 163-172.

Chung, D. H. and Buessem, W. R. (1967). The Voigt-Reuss-Hill approximation and elastic moduli of polycrystalline $\mathrm{MgO}, \mathrm{CaF}_{2}, \beta-\mathrm{ZnS}, \mathrm{ZnSe}$ and CdTe. J. Appl. Phys. 38, 2535-2540.

Chung, D. H. and Buessem, W. R. (1967). The elastic anisotropy of crystals. J. Appl. Phys. 38, 2010-2012.

Cullity, B. D. (1972). Introduction to Magnetic Materials. Addison-Wesley Publ. Reading.

Hill, R. (1952). The elastic behaviour of a crystalline aggregate. Proc. Phys. Soc. A65, 349-354.

Kopineck, H. J. (1986). On-Line Texture Measurement in a Production Line. In: Experimental Techniques of Texture Analysis. Ed. H. J. Bunge. DGM Informationsgesellschaft, Oberursel, 171-182.

Kopineck, H. J. and Bunge, H. J. (1988). A fixed angle texture analyzer for texture inspection and property control in sheet production lines. In: Directional Properties of Materials. Ed. H. J. Bunge DGM Informationsgesellschaft, Oberursel, 251-262.

Kopineck, H. J. and Otten, H. (1987). Texture Analyzer for On-line $r_{m}$-Value Estimation. Textures and Microstructures 7, 97-113.

Kröner, E. (1958). Berechnung der elastischen Konstanten des Vielkristalles aus den Konstanten des Einkristalles. Z. Phys. 151, 504-518.

Morris, P. R. (1969). Averaging fourth-rank tensors with weight functions. J. Appl. Phys. 40, 447-448. 
Reuss, A. (1929). Berechung der Fließgrenze von Mischkristallen auf Grund der Plastizitätsbedingung für Einkristalle. Z. Angew. Math. Mech. 9, 49-58.

Sakata, K., Daniel, D., and Jonas, J. J. (1989). Estimation of 4th and 6th Order ODF Coefficients From Elastic Properties in Cold Rolled Steel Sheets. Textures and Microstructures. 11, 41-56.

Voigt, W. (1928). Lehrbuch der Kristallphysik. B. G. Teubner Verlag, Leipzig.

Wassermann, G. and Grewen, J. (1962). Texturen metallischer Werkstoffe. Springer Verlag, Berlin. 